\title{
Analysis of the Kondo Resonance in a Single Quantum Dot Asymmetrically Coupled to Ferromagnetic Electrodes with Non-Collinear Magnetizations
}

\author{
M. WAWRZYNIAK* \\ Faculty of Physics, Adam Mickiewicz University \\ Umultowska 85, 61-614 Poznań, Poland
}

\begin{abstract}
The Kondo effect is studied theoretically in the framework of the non-equilibrium Green function formalism. The system under consideration consists of a single quantum dot asymmetrically coupled to ferromagnetic electrodes, whose magnetic moments are non-collinear. The spin-dependent density of states, as well as the transport characteristics such as differential conductance and tunneling magnetoresistance through the system are obtained using the equation of motion method. Numerical illustration of the mentioned quantities for several magnetic configurations and coupling strengths is presented and discussed.
\end{abstract}

PACS numbers: 72.15.Qm, 73.21.La, 73.23.Hk

\section{Introduction}

Theoretical analysis as well as experimental observation [1] of the Kondo effect in quantum dots have been intensively developed during last decade. The Kondo effect was studied theoretically first for nonmagnetic systems [2], then for magnetic systems with collinear magnetic configurations [3]. Recent works on the subject have been addressed mainly to the problem of transport in quantum dots attached symmetrically to magnetic leads in non-collinear configurations [4], but asymmetrical coupling has been also considered [5].

In this work we apply the equation of motion method for non-equilibrium Green function in order to analyze transport through quantum dot asymmetrically coupled to magnetic electrodes in non-collinear configuration.

\section{Model and method}

The system under consideration consist of a single level quantum dot attached to two ferromagnetic electrodes, whose magnetic moments are in general non-collinear. The system is described by the Hamiltonian of the general form

$$
H=H_{\mathrm{L}}+H_{\mathrm{R}}+H_{\mathrm{T}}+H_{\mathrm{D}},
$$

where $H_{\alpha}=\sum_{\boldsymbol{k} \beta} \epsilon_{\alpha \boldsymbol{k} \beta} a_{\alpha \boldsymbol{k} \beta}^{\dagger} a_{\alpha \boldsymbol{k} \beta}$ for $\alpha=\mathrm{L}, \mathrm{R}$ describes electrons in electrodes within noninteracting approximation. Term $H_{\mathrm{D}}$ describing the dot has the form

$$
H_{\mathrm{D}}=\sum_{\sigma} \epsilon_{\sigma} d_{\sigma}^{\dagger} d_{\sigma}+U d_{\uparrow}^{\dagger} d_{\uparrow} d_{\downarrow}^{\dagger} d_{\downarrow}
$$

where $\epsilon_{\sigma}$ is the energy of the dot level and $U$ is the
Coulomb repulsion parameter which is assumed to be finite. Tunneling events between the dot and electrodes are described by

$$
H_{\mathrm{T}}=\sum_{\boldsymbol{k} \alpha} \sum_{\beta \sigma} T_{\alpha \boldsymbol{k} \beta} a_{\alpha \boldsymbol{k} \beta}^{\dagger} R_{\alpha \beta \sigma} d_{\sigma}+\text { h.c., }
$$

where $T_{\alpha \boldsymbol{k} \beta}$ denote the tunneling matrix elements, whereas $R_{\alpha \beta \sigma}$ are elements of relevant spin rotation ma$\operatorname{trix} \boldsymbol{R}_{\alpha}$ :

$$
\boldsymbol{R}_{\alpha}=\left(\begin{array}{cc}
\cos \left(\phi_{\alpha} / 2\right) & -\sin \left(\phi_{\alpha} / 2\right) \\
\sin \left(\phi_{\alpha} / 2\right) & \cos \left(\phi_{\alpha} / 2\right)
\end{array}\right) .
$$

The angle $\phi_{\alpha}$ is the angle between the local quantization axis in the electrode and the global quantization axis on the dot. Within this paper we assume $-\phi_{\mathrm{L}}=\phi_{\mathrm{R}}=\phi / 2$. The coupling strength is described by $\Gamma_{\alpha \beta}(\epsilon)=2 \pi \sum_{\boldsymbol{k}}\left|T_{\alpha \boldsymbol{k} \beta}\right|^{2} \delta\left(\epsilon-\epsilon_{\alpha \boldsymbol{k} \beta}\right)$ and is assumed to be energy independent within the energy band extending from $-D$ to $D$, whereas $\Gamma_{\alpha \beta}(\epsilon)=\Gamma_{\alpha \beta}=\Gamma_{\alpha}^{0}\left(1 \pm p_{\alpha}\right)$, where $p_{\alpha}$ denotes spin polarization in the $\alpha$-th electrode. The coupling asymmetry parameter $\gamma$ has been introduced via $\Gamma_{\mathrm{R}}^{0}=\gamma \Gamma_{\mathrm{L}}^{0}$, for $1 \geq \gamma \geq 0$, which means that the system is coupled symmetrically for $\gamma=1$.

In order to calculate the spin-dependent density of states, electric current and tunneling magnetoresistance (TMR) the equation of motion technique for nonequilibrium Green function has been applied. The current flowing through the system has been calculated using formulae extended for non-collinear system, originally given by Meir et al. [2]. Within this procedure the lesser Green function as well as occupation numbers and renormalized dot level $\epsilon_{\sigma}$ have been calculated self-consistently.

*e-mail: laura.phys@wp.pl 


\section{Numerical results}

Spin-dependent density of states for the situation when the ferromagnetic electrodes are in antiparallel configuration for a few coupling asymmetry parameters $\gamma$ is shown in Fig. 1. It is well known that for symmetrically coupled quantum dot a single Kondo resonance is observed $[5,6]$. It is due to the fact that the exchange field, which results from an interaction between magnetic electrodes and spin on the dot, vanishes for $-\phi_{\mathrm{L}}=\phi_{\mathrm{R}}=\pi / 2$ and, as a consequence there is no spin-splitting of the dot level. Numerical illustration in Fig. 1 shows that splitting and suppression of the Kondo peak for the antiparallel magnetic configuration may be achieved by introducing coupling asymmetry. The suppression of the Kondo resonance increases with increasing asymmetry (decreasing $\gamma$ parameter).

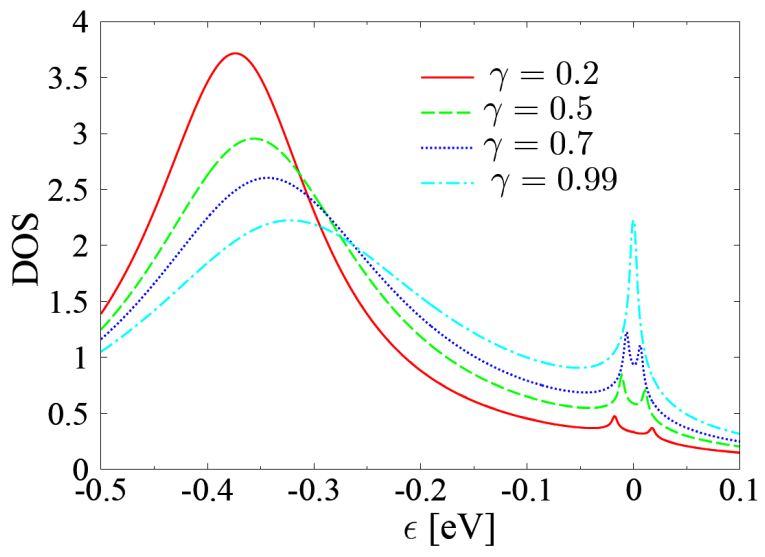

Fig. 1. Density of states of the quantum dot attached to magnetic leads in antiparallel configuration, calculated for a few asymmetry parameters $\gamma$ as indicated in the figure. The other parameters are: $\epsilon_{d}=-0.45 \mathrm{eV}$, $U=5 \mathrm{eV}, \Gamma_{0}=0.15 \mathrm{eV}, k_{\mathrm{B}} T=0.002 \mathrm{eV}, p_{\mathrm{L}}=$ $p_{\mathrm{R}}=0.2$ and electron band width extending from $-25 \mathrm{eV}$ to $25 \mathrm{eV}$.

In Fig. 2 the spin-dependent density of states for highly asymmetrically coupled quantum $\operatorname{dot}(\gamma=0.1)$ for a few magnetic configurations is presented. The spin-splitting of the Kondo resonance is clearly visible for all magnetic configurations and tends to decrease with changing configuration from parallel to antiparallel one. As mentioned above, this splitting is a result of non-zero exchange field occurring on the dot. Such spin-splitting exists also for the situation of the symmetrically coupled quantum dot when polarization of the left and right electrodes differs $\left(p_{\mathrm{L}} \neq p_{\mathrm{R}}\right)$, due to $\Gamma_{\alpha \beta}(\epsilon)=\Gamma_{\alpha \beta}=\Gamma_{\alpha}^{0}\left(1 \pm p_{\alpha}\right)$.

Numerical results for the corresponding differential conductance and TMR defined as

$$
\mathrm{TMR}=\frac{G_{\text {diff }}\left(\phi_{\mathrm{L}}=0, \phi_{\mathrm{R}}=0\right)-G_{\text {diff }}\left(\phi_{\mathrm{L}}, \phi_{\mathrm{R}}\right)}{G_{\text {diff }}\left(\phi_{\mathrm{L}}, \phi_{\mathrm{R}}\right)},
$$

vs. bias voltage are shown in Fig. 3 for several values of $\phi$. One may observe the splitting of the Kondo anomaly in the differential conductance for all magnetic configura-

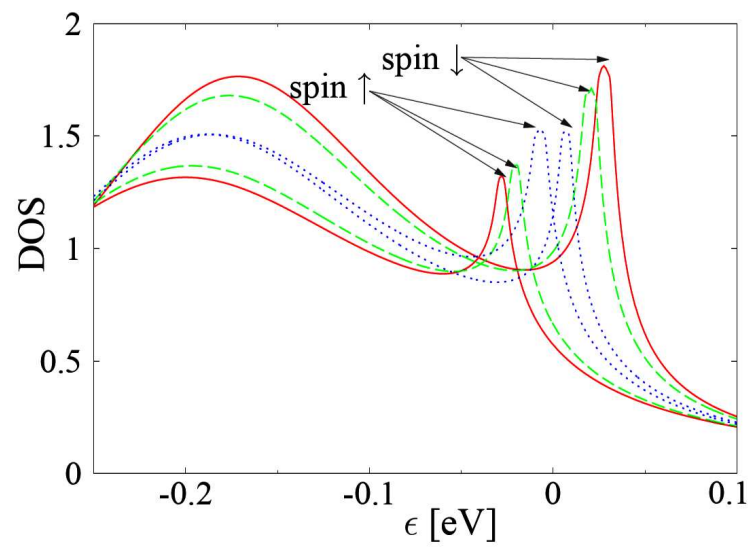

Fig. 2. Density of states calculated for different magnetic configurations: $\phi=0$ (solid line), $\phi=\pi / 2$ (dashed line) and $\phi=\pi$ (dotted line) and parameters: $\gamma=0.1$, $\epsilon_{d}=-0.3 \mathrm{eV}, U=5 \mathrm{eV}, \Gamma_{0}=0.2 \mathrm{eV}, k_{\mathrm{B}} T=0.001 \mathrm{eV}$, $p_{\mathrm{L}}=0.1, p_{\mathrm{R}}=0.6$, electron band width extending from $-25 \mathrm{eV}$ to $25 \mathrm{eV}$.

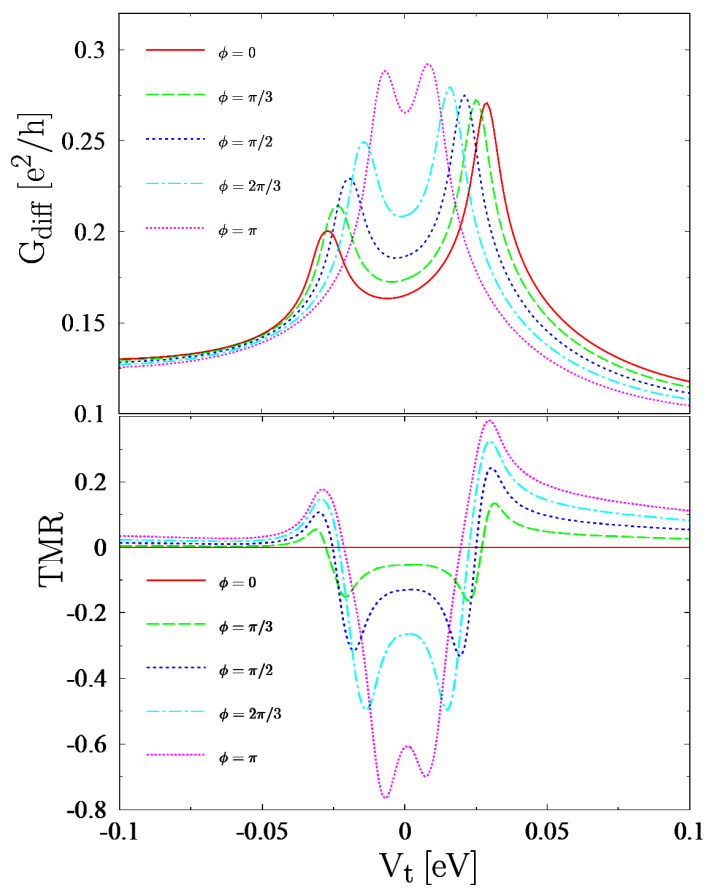

Fig. 3. Differential conductance (upper) and TMR (lower) calculated for parameters as in Fig. 2 and for magnetic configurations as indicated in the figure.

tions. Moreover, the Kondo peaks in differential conductance are asymmetrical with respect to the bias reversal. This asymmetry is also visible in TMR.

\section{Acknowledgments}

This work has been supported by Polish Ministry of Science and Higher Education as a research project in years 2006-2008. 


\section{References}

[1] D. Goldhaber-Gordon, H. Shtrikman, D. Mahalu, D. Abush-Magder, U. Meirav, M.A. Kastner, Nature 391, 156 (1998).

[2] Y. Meir, P.S. Wingreen, P.A. Lee, Phys. Rev. Lett. 70, 2601 (1993).

[3] J. Martinek, Y. Utsumi, H. Immamura, J. Barnaś, S. Maekawa, J. Koenig, G. Schoen, Phys. Rev. Lett. 91, 127203 (2003).
[4] N. Sergueev, Q. Sun, H. Guo, B.G. Wang, J. Wang, Phys. Rev. B 65, 165303 (2002).

[5] R. Świrkowicz, M. Wilczyński, M. Wawrzyniak, J. Barnaś, Phys. Rev. B 73, 193312 (2006).

[6] J. Martinek, Y. Utsumi, H. Imamura, J. Barnaś, S. Maekawa, J. Koenig, G. Schoen, Phys. Rev. Lett. 91, 127203 (2003). 